\title{
Comparing Outcomes of Two Antiviral Therapy Combinations among COVID-19 Patients
}

\author{
Hossein Mazaherpour $\mathbb{D D}^{1},{ }^{1}$ Masoomeh Sofian $(\mathbb{D}){ }^{2}$ Elham Farahani ${ }^{(D)},{ }^{1}$ Alireza Abdi $\left(\mathbb{D},{ }^{3}\right.$ \\ Sakine Mazaherpour $\left(\mathbb{D},{ }^{4}\right.$ Anahita Bavand $\left(\mathbb{D},{ }^{5}\right.$ and Amitis Ramezani ${ }^{5}{ }^{5}$ \\ ${ }^{1}$ Arak University of Medical Sciences, Arak, Iran \\ ${ }^{2}$ Infectious Diseases Research Center, Arak University of Medical Sciences, Arak, Iran \\ ${ }^{3}$ Kermanshah University of Medical Sciences, Kermanshah, Iran \\ ${ }^{4}$ Treatment Management of Social Security Organization of Khuzestan Province, Mahshahr, Iran \\ ${ }^{5}$ Clinical Research Department, Pasteur Institute of Iran, Tehran, Iran
}

Correspondence should be addressed to Masoomeh Sofian; dr.sofian@arakmu.ac.ir and Amitis Ramezani; amitisramezani@hotmail.com

Received 26 September 2021; Accepted 8 December 2021; Published 7 January 2022

Academic Editor: Walid Saibi

Copyright (c) 2022 Hossein Mazaherpour et al. This is an open access article distributed under the Creative Commons Attribution License, which permits unrestricted use, distribution, and reproduction in any medium, provided the original work is properly cited.

\begin{abstract}
Several therapeutic regimens for COVID-19 have been studied, such as combination antiviral therapies. We aimed to compare outcome of two types of combination therapies atazanavir/ritonavir (ATV/r) or lopinavir/ritonavir (LPV/r) plus hydroxychloroquine among COVID-19 patients. 108 patients with moderate and severe forms of COVID-19 were divided into two groups (each group 54 patients). One group received ATV/r plus hydroxychloroquine, and the other group received hydroxychloroquine plus LPV/r. Then, both groups were evaluated and compared for clinical symptoms, recovery rates, and complications of treatment regimens. Our findings showed a significant increase in bilirubin in ATV/r-receiving group compared to LPV/r receivers. There was also a significant increase in arrhythmias in the LPV/r group compared to the ATV/r group during treatment. Other findings including length of hospital stay, outcome, and treatment complications were not statistically significant. There is no significant difference between protease inhibitor drugs including ATV/r and LPV/r in the treatment of COVID-19 regarding clinical outcomes. However, some side effects such as hyperbilirubinemia and arrhythmia were significantly different by application of atazanavir or lopinavir.
\end{abstract}

\section{Introduction}

Coronavirus Disease 2019 (COVID-19) started in late 2019 in Wuhan, China, which then spread rapidly to many countries worldwide $[1,2]$. This pandemic disease has infected more than 200 million people worldwide $[2,3]$ caused by a single-stranded RNA virus with human and animal hosts [4-6]. The average incubation period of the disease is between 4 and 5 days, but it may last up to 14 days. Some data show that SARS-CoV-2 with mild presentation leads to short protection rather than severe infection [5, 7-9]. The disease may differ from a mild and asymptomatic form to a severe presentation with acute respiratory distress syndrome (ARDS) and death. According to the results of the studies, about $81 \%$ of patients presented moderate and mild forms whereas $14 \%$ and $5 \%$ had severe and threatening to fatal forms of the disease, respectively [10-12]. Symptoms including fever, cough, and dyspnea have been found in about $70 \%$ whereas muscle pain and headache were seen in $36 \%$ and $34 \%$, respectively [13].

Several therapeutics and vaccines have been investigated to overcome COVID-19 disease [6, 14]. Among these drugs, protease inhibitors which are used for treatment of HIV-1 are applied in COVID-19 [15]. Protease inhibitors impair the virus replication by inhibition of protease, which are hepatically metabolized via the CYP isoenzyme CYP3A4. Lopinavir/ritonavir (LPV/r) is a potent inhibitor of CYP3A4 metabolism; therefore, it induces more drug interactions 
[16]. Moreover, LPV/r, as a protease inhibitor drug, can inhibit the protease 3CLpro enzyme, which is one of the RNA polymerase-dependent proteases of the virus and is involved in virus replication [15]. Atazanavir/ritonavir $(\mathrm{ATV} / \mathrm{r})$ is another drug belonging to the protease inhibitor family that has a greater inhibitory effect on CYP3A4 and fewer side effects compared to LPV/r; therefore, ATV/r is easier to tolerate for patients. Moreover, LPV/r has been shown to have better pulmonary permeability [17]. COVID-19 causes a widespread inflammatory response in the body through the cytokine storm mediated by interleukin-6. Some symptoms of COVID-19 stem from this inflammatory reaction. Evidence showed that some protease inhibitors such as atazanavir can reduce symptoms and disease severity to some extent by decreasing the release of interleukin-6 from primary monocytes $[18,19]$.

Protease inhibitor drugs cause a number of side effects. This includes common side effects of $\mathrm{LPV} / \mathrm{r}$ as nausea, vomiting, diarrhea, hepatotoxicity, prolonged Q-T on electrocardiogram (ECG), skin rash, hyperlipidemia, and abdominal pain. Furthermore, ATV/r can cause some side effects such as hyperbilirubinemia, rash, fever, nausea, vomiting, diarrhea, high cholesterol, and cough. What is more, hyperbilirubinemia is the most frequent adverse effect of ATV. ATV can cause a reversible, dose-dependent increase predominantly of unconjugated bilirubin [20].

In previous studies, hydroxychloroquine caused some complications such as hemolysis in patients with glucose6-phosphate dehydrogenase (G6PD) deficiency, bone marrow suppression, cardiomyopathy, some abnormalities in ECG including prolonged PR, QRS, QT interval, and some new-onset cardiac arrhythmias such as bundle branching block (BBB) and atrioventricular block [21, 22]. On the other hand, the definite treatment of COVID-19 is not exactly known and the role and effectiveness of combination therapy in this disease are still a matter to be investigated.

In the present study, we aimed to compare the efficacy and side effects of two combination therapies including atazanavir/ritonavir $(\mathrm{ATV} / \mathrm{r})$ or lopinavir/ritonavir $(\mathrm{LPV} / \mathrm{r})$ plus hydroxychloroquine among COVID-19 patients.

\section{Methods}

2.1. Study Design and Participants. The study was designed as a clinical trial in which the patients who presented moderate or severe COVID-19 disease were recruited based on the diagnosis of the infectious disease specialist. Overall, 108 eligible patients with COVID-19 who were admitted to Ayatollah Khansari Hospital and Amir Al-Momenin Hospital in Arak city were investigated from May to October 2020.

Patients were divided into two groups $(n=54)$. Inclusion criteria were patients with moderate to severe COVID-19 that were hospitalized in the infectious and COVID wards of the hospital besides signing the informed consent. Exclusion criteria were the critical type of the disease that required intensive care unit (ICU) admission and intubation, a mild type of the disease without need to hospitalization, and unwillingness to participate in this study. Moreover, discharge was based on all of the following: (1) improvement in clinical signs and symptoms based on physician's opinion, (2) afebrile status for $72 \mathrm{~h}$ without antipyretics, and (3) saturation of peripheral oxygen $(\mathrm{SpO} 2)$ more than $93 \%$ in ambient air without supplemental oxygen.

In the present study, moderate and severe COVID-19 were defined according to WHO guidelines. Therefore, moderate form of COVID-19 is defined as the existence of primary symptoms of pneumonia (dyspnea, cough, and fever) and $\mathrm{SpO} 2 \geq 90 \%$ in ambient air and severe form is defined as profound pneumonia and $\mathrm{SpO} 2<90 \%$. The diagnosis approach was based on the WHO guideline so that positive reverse transcriptase-polymerase chain reaction (RT-PCR) of nasopharyngeal and oropharyngeal secretions or clinical manifestations plus findings of chest CT scan was highly suggestive for COVID-19 [7, 13].

This study was approved by the Ethics Committee of Arak University of Medical Sciences (approval ID: IR-ARAKMU.REC.1399.006). The study protocol was also registered as IRCT20200517047485N1. Written informed consents were obtained from the patients or one of the first-degree family members if the patient was unconscious.

2.2. Therapeutic Regimens. Patients of one group received hydroxychloroquine tablet $400 \mathrm{mg}$ single dose on the first day and LPV/r (200 mg/50 mg tablet) 2 tablets every 12 hours from the second day for at least 5-7 days.

The other group received hydroxychloroquine $200 \mathrm{mg}$ every 12 hours plus ATV/r 1 tablet $(300 \mathrm{mg} / 100 \mathrm{mg})$ daily for at least 5-7days. According to physician's judgment, the treatment period was longer whenever needed. The duration of treatment was at least 7 days. In addition, more treatments were also recorded such as other antiviral drugs, corticosteroids, intravenous immunoglobulin (IVIG), vitamin $\mathrm{C}$, antibiotics, analgesic agents, antinausea and vomiting agents, cardiovascular drugs, deep vein thrombosis (DVT), and stress ulcer prophylaxis.

2.3. Laboratory Tests. Demographic data, clinical presentations, underlying diseases, drug history, and laboratory and medical data were collected and documented. Clinical symptoms such as fever, sore throat, dyspnea, abdominal pain, and clinical signs including $\mathrm{SpO} 2$, pulse rate, respiratory rate, blood pressure, and temperature were recorded at the time of hospital admission. Vital signs and laboratory tests such as white blood cells (WBC), serum electrolytes, liver and kidney enzymes, inflammatory biomarkers consisting of C-reactive protein (CRP), and erythrocyte sedimentation rate (ESR) were extracted from the hospital information system (HIS) and patient's file every day and recorded in questionnaire.

During the hospitalization, the patients were examined daily for the presence or absence of signs and symptoms of COVID-19 disease as well as vital signs and daily laboratory tests which were collected through a questionnaire. The patient's ECG was taken daily or every other day upon admission and during the hospitalization. They were then interpreted by a cardiologist for the presence or absence of arrhythmia who was not aware of the patient's medication. 
2.4. Statistical Analysis. The statistical analysis was performed using SPSS version 23.0. A $p$ value less than 0.05 is considered statistically significant. Descriptive data were calculated as frequency, frequency percentage, and analytical statistics through the chi-square test.

\section{Results}

This study was performed on 108 patients with COVID-19 who were in the two groups of 54 patients. $47.2 \%$ of patients were male and $52.8 \%$ were female. The majority of patients aged from 60 to 79 years $(42.6 \%)$. The most common symptoms in both groups were dry cough (67.6\%), myalgia (65\%), and dyspnea (60.2\%), and the least common symptom was skin rash $(8.3 \%)$.

On admission, $27.8 \%$ of patients had $\mathrm{SpO} 2>94 \%$, and $41.7 \%$ of them had SpO2 between 90 and $94 \%$ and $30.6 \%$ had $\mathrm{SpO} 2$ less than $90 \%$. There was no significant difference between the two groups for the findings of physical examination at the time of admission including fever, blood pressure, respiratory rate, $\mathrm{SpO} 2$, and heart rate.

Based on the findings of physical examination and evaluation of patients during the treatment period (Table 1), the number of days with fever $(p=1.00)$, number of days with hypoxia $(p=0.343)$, number of days with cough $(p=0.334$ ) and myalgia $(p=0.412)$, and also the length of stay $(p=0.479)$ were not significantly different between the two groups.

Other findings include the number of days with dyspnea $(p=0.417)$, number of days with headache $(p=0.647)$, anorexia $(p=0.90)$, nausea and vomiting $(p=1.00)$, and diarrhea $(p=0.315)$.

Furthermore, sore throat $(p=1.00)$, abdominal pain $(p=0.69)$, and skin lesions $(p=0.08)$ assessed daily for all patients in the course of treatment were not significantly different between the two groups.

On the first day of hospitalization, laboratory tests such as complete blood count (CBC), ESR, CRP, liver enzymes, bilirubin, lipid profile, serum lactate dehydrogenase (LDH) and creatinine phosphokinase (CPK), and international normalized ratio (INR) were checked and were repeated during hospitalization according to the physician's opinion and existing instructions.

In addition, as each treatment regimen can lead to changes in these tests, the trend of changes was categorized as unchanged, ascending, and descending according to the patient's baseline test and the normal range for these tests (Table 2).

Laboratory test findings did not show any significant differences between two groups associated to values of white blood cell (WBC) $(p=0.127)$, hemoglobin $(p=0.554)$, platelets $(p=0.906)$, and lymphocyte percentage $(p=0.478)$ (Table 2). What is more, there was no significant difference for trend of ESR, CRP, creatinine, and liver enzymes (Table 2).

As a highlighted outcome, bilirubin was significantly higher in the group of patients who received ATV/r than the group of patients who received LPV/r $(p<0.001)$.
Hyperbilirubinemia is one of the most common side effects of $\mathrm{ATV} / \mathrm{r}$, and it is less common for $\mathrm{LPV} / \mathrm{r}$.

The other laboratory findings such as lipid profile, blood sugar, $\mathrm{CPK}, \mathrm{LDH}$, and INR were not significantly different.

The other significant result was a higher rate of arrhythmia in the LPV/r group than the ATV/r group $(p=0.019)$ (Table 3). In this study, 44 patients suffered arrhythmias during treatment period including 28 patients of the LPV/r group and 16 patients of the ATV/r group. The types of arrhythmias for these patients were sinus bradycardia $(n=10)$, prolonged Q-T $(n=9)$, sinus tachycardia $(n=7)$, first-degree block $(n=6)$, left bundle branch block (RBBB) $(n=6)$, right bundle branch block $(\mathrm{RBBB})(n=3)$, premature ventricular contraction (PVC) $(n=2)$, and premature atrial contraction (PAC) $(n=1)$. These arrhythmias were diagnosed by a cardiologist by comparing the initial ECG in admission day ECG of hospitalization days.

The treatment outcomes were classified as discharge with a good and stable general condition, evidence of complications during the hospital stay that continues until discharge or mortality. There was no significant difference in treatment outcomes between the two groups.

Unfortunately, five investigated patients including 4 females and 1 male (aged from 60 to 80) expired, from whom three patients belonged to the LPV/r group and the two were in the ATV/r group. All these patients had $\mathrm{SpO} 2$ less than $90 \%$ on admission. Moreover, one had no history of specific underlying disease whereas two had a history of diabetes and hypertension, one with a history of hypertension and coronary heart disease, and the last one with only a history of diabetes.

Our findings indicated that ten patients of the LPV/r group had complications during hospitalization and discharge. In addition, four patients had no increase in SpO2 and were dependent on oxygen on discharge, two patients had DVT, one had pulmonary thromboembolism (PTE), two had pressure ulcer, and one patient developed hemoptysis.

Finally, among the ATV/r group, even patients developed complications during hospitalization and on discharge. Three patients did not have increased SpO2 during hospitalization and on discharge, one patient developed pancreatitis, another had hemoptysis, and one showed gastrointestinal bleeding (GIB).

\section{Discussion}

To the best of our knowledge, in a few studies, outcomes and complications of combination therapy were compared among COVID-19 hospitalized patients. In our clinical trial, the efficacy and side effects of two combination therapies with $\mathrm{LPV} / \mathrm{r}$ and ATV/r plus hydroxychloroquine were compared between the two groups of COVID-19 patients.

The results of this study indicated that combination therapy with these drugs was not significantly different between two groups in terms of hospitalization length, complications, mortality rate following treatment period, time of alleviation from clinical symptoms, and clinical outcomes. 
TABLE 1: Comparison of clinical presentations in COVID-19 patients on two different combination therapies.

\begin{tabular}{|c|c|c|c|c|}
\hline & Duration (days) & $\begin{array}{c}\text { Lopinavir/ritonavir } \\
N(\%)\end{array}$ & $\begin{array}{c}\text { Atazanavir/ritonavir } \\
N(\%) \\
\end{array}$ & $p$ value \\
\hline \multirow{2}{*}{ Febrile } & $0-3$ & $53(50)$ & $53(50)$ & \multirow{2}{*}{1.00} \\
\hline & $3-5$ & $1(50)$ & $1(50)$ & \\
\hline \multirow{6}{*}{ Admission } & $3-5$ & $0(0)$ & $1(100)$ & \multirow{6}{*}{0.479} \\
\hline & $5-7$ & $6(42.9)$ & $8(57.1)$ & \\
\hline & $7-9$ & $23(53.5)$ & $20(46.5)$ & \\
\hline & $9-11$ & $21(56.8)$ & $16(43.2)$ & \\
\hline & $11-13$ & $4(33.3)$ & $8(66.7)$ & \\
\hline & $>14$ & $0(0)$ & $1(100)$ & \\
\hline \multirow{6}{*}{ Hypoxic } & $0-3$ & $23(43.4)$ & $30(56.6)$ & \multirow{6}{*}{0.343} \\
\hline & $3-5$ & $18(62.1)$ & $11(37.9)$ & \\
\hline & $5-7$ & $1(20)$ & $4(80)$ & \\
\hline & $7-9$ & $1(33.3)$ & $2(66.7)$ & \\
\hline & $9-11$ & $8(61.5)$ & $5(38.5)$ & \\
\hline & $11-13$ & $3(60)$ & $2(40)$ & \\
\hline \multirow{3}{*}{ Myalgia } & $0-5$ & $48(52.2)$ & $44(47.8)$ & \multirow{3}{*}{0.412} \\
\hline & $5-10$ & $6(40)$ & $9(60)$ & \\
\hline & $>10$ & $0(0)$ & $1(100)$ & \\
\hline \multirow{3}{*}{ Cough } & $0-5$ & $39(47.6)$ & $43(52.4)$ & \multirow{3}{*}{0.334} \\
\hline & $5-10$ & $15(60)$ & $10(40)$ & \\
\hline & $>10$ & $0(0)$ & $1(100)$ & \\
\hline \multirow{3}{*}{ Dyspnea } & $0-5$ & $47(52.8)$ & $42(47.2)$ & \multirow{3}{*}{0.417} \\
\hline & $5-10$ & $6(35.3)$ & $11(64.7)$ & \\
\hline & $>10$ & $1(50)$ & $1(50)$ & \\
\hline \multirow{2}{*}{ Headache } & $0-5$ & $51(49.5)$ & $52(50.5)$ & \multirow{2}{*}{0.647} \\
\hline & $5-10$ & $3(60)$ & $2(40)$ & \\
\hline \multirow{2}{*}{ Anorexia } & $0-5$ & $51(50.5)$ & $50(49.5)$ & \multirow{2}{*}{0.90} \\
\hline & $5-10$ & $2(40)$ & $3(60)$ & \\
\hline \multirow{2}{*}{ Nausea } & $0-5$ & $53(50)$ & $53(50)$ & \multirow{2}{*}{1.00} \\
\hline & $5-10$ & $1(50)$ & $1(50)$ & \\
\hline \multirow{2}{*}{ Diarrhea } & $0-5$ & $53(49.5)$ & $54(50.5)$ & \multirow{2}{*}{0.315} \\
\hline & $5-10$ & $1(100)$ & $0(0)$ & \\
\hline \multirow{2}{*}{ Sore throat } & $0-5$ & $53(50)$ & $53(50)$ & \multirow{2}{*}{1.00} \\
\hline & $5-10$ & $1(50)$ & $1(50)$ & \\
\hline Abdominal pain & $0-5$ & $3(42.9)$ & $4(57.1)$ & 0.696 \\
\hline Skin rash & $0-5$ & $2(22.2)$ & $7(77.8)$ & 0.082 \\
\hline
\end{tabular}

The rate of hyperbilirubinemia was significantly higher in the $\mathrm{ATV} / \mathrm{r}$ group than the LPV/r group. In this study, $85.4 \%$ of patients that received ATV/r had hyperbilirubinemia. In line with our study, a meta-analysis on the clinical benefit of ATV/r- and LPV/r-based cART in HIV patients demonstrated that the risk of ATV/r-induced hyperbilirubinemia is very high in comparison with LPV/r [16].

Atazanavir-induced hyperbilirubinemia is indirect hyperbilirubinemia that is created by inhibition of the UDP-glucuronyltransferase (UGT) enzyme that conjugates bilirubin, which is associated with genetic characteristics [23]. Moreover, it has been previously reported that $\mathrm{UGT} 1 \mathrm{~A} 1 * 28$ polymorphism increased the risk of atazanavir-induced hyperbilirubinemia in HIV patients
[16]. Therefore, the use of pharmacogenetics tests can help to determine a patient's susceptibility to drug toxicity and to facilitate the selection of appropriate medicine.

The results of some studies revealed that boosted atazanavir with ritonavir increases the risk of indirect hyperbilirubinemia compared to atazanavir alone [24, 25].

In a study by Malan et al. which was performed on 400 patients, the rate of hyperbilirubinemia was $59 \%$ in the $\mathrm{ATV} / \mathrm{r}$ group and $20 \%$ in the atazanavir group [24].

Interestingly, Moyle $\mathrm{G}$ et al.'s study revealed a meaningful decrease in unconjugated and total bilirubin following zinc sulfate $\left(\mathrm{ZnSO}_{4}\right)$ intake and a limited decrease in ATV/ $\mathrm{r}$ plasma exposure suggesting $\mathrm{ZnSO}_{4}$ supplementation may represent a useful medicine in the short-term management 
TABLE 2: Comparison of laboratory findings between COVID-19 cases receiving two types of therapeutics.

\begin{tabular}{|c|c|c|c|c|}
\hline Features & Trend & $\begin{array}{c}\text { Lopinavir/ritonavir } \\
N(\%)\end{array}$ & $\begin{array}{c}\text { Atazanavir/ritonavir } \\
N(\%)\end{array}$ & $p$ value \\
\hline \multirow{3}{*}{ WBC on admission } & No change & $42(55.3)$ & $34(44.7)$ & \multirow{3}{*}{0.127} \\
\hline & Increasing & $11(42.3)$ & $15(57.7)$ & \\
\hline & Decreasing & $1(16.7)$ & $5(83.3)$ & \\
\hline \multirow{3}{*}{$\mathrm{Hb}$} & No change & $51(51)$ & $49(49)$ & \multirow{3}{*}{0.554} \\
\hline & Increasing & $0(0)$ & $1(100)$ & \\
\hline & Decreasing & $3(42.9)$ & $4(51.7)$ & \\
\hline \multirow{3}{*}{ Platelet } & No change & $35(48.6)$ & $37(51.4)$ & \multirow{3}{*}{0.906} \\
\hline & Increasing & $4(50)$ & $4(50)$ & \\
\hline & Decreasing & $15(53.6)$ & $13(46.4)$ & \\
\hline \multirow{3}{*}{ Lymphocyte count } & No change & $37(54.4)$ & $31(45.6)$ & \multirow{3}{*}{0.478} \\
\hline & Increasing & $16(41.2)$ & $26(57.9)$ & \\
\hline & Decreasing & $1(50)$ & $1(50)$ & \\
\hline \multirow{3}{*}{ ESR } & No change & $19(46.3)$ & $22(53.7)$ & \multirow{3}{*}{0.836} \\
\hline & Increasing & $34(52.3)$ & $31(47.7)$ & \\
\hline & Decreasing & $1(50)$ & $1(50)$ & \\
\hline \multirow{3}{*}{ CRP } & No change & $21(50)$ & $21(50)$ & \multirow{3}{*}{0.587} \\
\hline & Increasing & $32(51.6)$ & $30(48.4)$ & \\
\hline & Decreasing & $1(25)$ & $3(75)$ & \\
\hline \multirow{2}{*}{ Creatinine } & No change & $42(50.6)$ & $41(49.4)$ & \multirow{2}{*}{0.82} \\
\hline & Increasing & $12(48)$ & $13(52)$ & \\
\hline \multirow{3}{*}{ AST } & No change & $36(49.3)$ & $37(50.7)$ & \multirow{3}{*}{0.978} \\
\hline & Increasing & $17(51.5)$ & $16(48.5)$ & \\
\hline & Decreasing & $1(50)$ & $1(50)$ & \\
\hline \multirow{3}{*}{ ALT } & No change & $42(53.8)$ & $36(46.2)$ & \multirow{3}{*}{0.423} \\
\hline & Increasing & $11(40.7)$ & $16(59.3)$ & \\
\hline & Decreasing & $1(33.3)$ & $2(66.7)$ & \\
\hline \multirow{3}{*}{ ALP } & No change & $47(48.5)$ & $50(51.5)$ & \multirow{3}{*}{0.579} \\
\hline & Increasing & $6(66.7)$ & $3(33.3)$ & \\
\hline & Decreasing & $1(50)$ & $1(50)$ & \\
\hline \multirow{2}{*}{ Bilirubin } & No change & $47(78.3)$ & $13(21.7)$ & \multirow{2}{*}{$<0.001$} \\
\hline & Increasing & $7(14.6)$ & $41(85.4)$ & \\
\hline \multirow{2}{*}{ Lipid profile } & No change & $38(47.5)$ & $42(52.5)$ & \multirow{2}{*}{0.380} \\
\hline & Increasing & $16(57.1)$ & $12(42.9)$ & \\
\hline \multirow{2}{*}{ BS } & No change & $41(53.2)$ & $36(46.8)$ & \\
\hline & Increasing & $13(41.9)$ & $18(58.1)$ & 0.288 \\
\hline & No change & $36(49.3)$ & $37(50.7)$ & \\
\hline $\mathrm{CPK}$ & Increasing & $17(53.1)$ & $15(46.9)$ & 0.790 \\
\hline & Decreasing & $1(33.3)$ & $2(66.7)$ & \\
\hline & No change & $37(50.7)$ & $36(49.3)$ & \\
\hline $\mathrm{LDH}$ & Increasing & $16(50)$ & $16(50)$ & 0.841 \\
\hline & Decreasing & $1(33.3)$ & $2(66.7)$ & \\
\hline IND & No change & $50(52.6)$ & $45(47.4)$ & 0120 \\
\hline $\mathrm{INR}$ & Increasing & $4(30.8)$ & $9(69.2)$ & 0.139 \\
\hline
\end{tabular}

ALP: alkaline phosphatase; ALT: alanine amino transaminase; AST: aspartate amino transaminase; BS: blood sugar; CBC: complete blood count; CPK: creatinine phosphokinase; CRP: C-reactive protein; ESR: erythrocyte sedimentation rate; Hb: hemoglobin; INR: international normalized ratio; LDH: lactate dehydrogenase; WBC: white blood cell. Bold $p$ value indicates statistical significance. 
TABLE 3: The outcome of two different combination therapies among COVID-19 patients.

\begin{tabular}{|c|c|c|c|c|}
\hline & & $\begin{array}{c}\text { Lopinavir/ritonavir } \\
N(\%)\end{array}$ & $\begin{array}{c}\text { Atazanavir/ritonavir } \\
N(\%)\end{array}$ & $p$ value \\
\hline \multirow[t]{2}{*}{ Arrhythmia } & & $28(63.6)$ & $16(36.4)$ & 0.019 \\
\hline & Discharge & $41(47.7)$ & $45(52.3)$ & \\
\hline \multirow[t]{2}{*}{ End of treatment } & Complication & $10(58.8)$ & $7(41.20)$ & 0.633 \\
\hline & Expired & $3(60)$ & $2(40)$ & \\
\hline
\end{tabular}

Bold $p$ value indicates statistical significance.

of ATV-related hyperbilirubinemia in HIV-infected patients [16]. Therefore, further studies are needed to evaluate the beneficial effects of some supplements in combination with $\mathrm{ATV} / \mathrm{r}$ to decrease its side effect.

The cardiac arrhythmias rate during the treatment period was significantly higher in the LPV/r group compared to the ATV/r group. In this study, almost all the patients received antibiotic therapy in addition to antiviral therapy including fluoroquinolones and macrolides which may cause arrhythmias. On the other hand, the interaction of LPV/r with these antibiotics, as well as with hydroxychloroquine, which has been used as part of combination therapy against COVID-19 in these patients, might be the cause of higher arrhythmias in the LPV/r group.

Anson et al. showed that LPV/r could predispose patients to arrhythmias such as prolonged Q-T and torsade de point by inhibiting of human ether-a-go-go-related gene (HERG) potassium channels and potassium current (IKr) channels [16]. In Bessière et al.'s study, combination therapy with hydroxychloroquine and azithromycin caused prolonged Q-T in $10.7-36 \%$ of patients [16].

Russo et al.'s findings indicated that $23 \%$ of patients developed arrhythmias following the COVID-19 treatment by hydroxychloroquine and azithromycin [26].

\section{Conclusions}

In this study, two different combination therapies (ATV/r and LPV/r group) in terms of efficacy and side effects were comparatively investigated. The findings indicated a significant increase in bilirubin in ATV/r receivers compared to LPV/r-treated patients. Arrhythmias were also significantly increased in the LPV/r group compared to the ATV/r group during the treatment. Apart from the rate of hyperbilirubinemia and cardiac arrhythmia, other indices were not statistically various between the two groups. There was no significant difference for mortality rate and complications of treatment regimen between two groups. It seems that the current applying agents are not preferably acceptable to overcome COVID-19 infection, and therefore, more therapeutic antiviral regimens must be studied in this era.

\section{Abbreviations}

ALP: Alkaline phosphatase

ALT: Alanine amino transaminase

AST: Aspartate amino transaminase

BS: $\quad$ Blood sugar
CBC: Complete blood count

CPK: Creatinine phosphokinase

CRP: C-reactive protein

ECG: Electrocardiogram

ESR: Erythrocyte sedimentation rate

GIB: Gastrointestinal bleeding

$\mathrm{Hb}$ : Hemoglobin

INR: International normalized ratio

LDH: Lactate dehydrogenase

PAC: Premature atrial contraction

PTE: Pulmonary thromboembolism

RBBB: Right bundle branch block

SpO2: Saturation of peripheral oxygen

WBC: White blood cell.

\section{Data Availability}

The data that support the findings of this study are available from the corresponding author upon reasonable request.

\section{Conflicts of Interest}

The authors declare no competing interests.

\section{Authors' Contributions}

H.M., as a principal investigator of the study, designed the study. M.S., E.F., and A.A. cooperated in visiting patients and project implementation. S.M. collected and analyzed the data. A.B. wrote the draft of the manuscript. A.R. supervised, revised, and finalized the manuscript. All authors read and approved the manuscript.

\section{Acknowledgments}

We would like to thank the medical teams of Ayatollah Khansari Hospital and Amir Al-Momenin Hospital for their nice cooperation. This study was supported by Arak University of Medical Sciences, Arak, Iran (Grant number 3619).

\section{References}

[1] Coronavirus disease 2019 (COVID-19): cases in US, "Centers for Disease Control and Prevention,” 2020, https://www.cdc .gov/coronavirus/2019-ncov/cases-updates/cases-inus.html.

[2] World Health Organization, "World Health Organization," 2020, https://www.who.int/emergencies/diseasescoronavirus2019/novel-/situationreports/. 
[3] H. Rahmani, E. Davoudi-Monfared, A. Nourian et al., "Comparing outcomes of hospitalized patients with moderate and severe COVID-19 following treatment with hydroxychloroquine plus atazanavir/ritonavir," DARU Journal of Pharmaceutical Sciences., vol. 28, no. 2, pp. 625-634, 2020.

[4] J. Cui, F. Li, and Z.-L. Shi, "Origin and evolution of pathogenic coronaviruses," Nature Reviews. Microbiology, vol. 17, no. 3, pp. 181-192, 2019.

[5] M. Sofian, A. A. Velayati, M. Banifazl et al., "SARS-CoV-2, a virus with many faces: a series of cases with prolonged persistence of COVID-19 symptoms," Wiener Medizinische Wochenschrift, vol. 171, no. 1-2, pp. 3-6, 2021.

[6] S. M. Sadat, M. R. Aghadadeghi, M. Yousefi, A. Khodaei, M. Sadat Larijani, and G. Bahramali, "Bioinformatics analysis of SARS-CoV-2 to approach an effective vaccine candidate against COVID-19," Molecular Biotechnology, vol. 63, no. 5, pp. 389-409, 2021.

[7] W.-j. Guan, Z.-y. Ni, Y. Hu et al., "Clinical characteristics of coronavirus disease 2019 in China," New England Journal of Medicine, vol. 382, no. 18, pp. 1708-1720, 2020.

[8] M. Salehi-Vaziri, M. D. Omrani, M. H. Pouriayevali et al., "SARS-CoV-2 presented moderately during two episodes of the infection with lack of antibody responses," Virus Research, vol. 299, p. 198421, 2021.

[9] M. Salehi-Vaziri, M. H. Pouriayevali, F. Fotouhi et al., "SARSCoV-2 re-infection rate in Iranian COVID-19 cases within one-year follow- up," Microbial Pathogenesis, vol. 161, Partt B, p. 105296, 2021.

[10] M. Salehi-Vaziri, T. Jalali, B. Farahmand et al., "Clinical characteristics of SARS-CoV-2 by re-infection vs. reactivation: a case series from Iran," European Journal of Clinical Microbiology \& Infectious Diseases, vol. 40, no. 8, pp. 1713-1719, 2021.

[11] Z. Wu and J. M. McGoogan, "Characteristics of and important lessons from the coronavirus disease 2019 (COVID-19) outbreak in China: summary of a report of 72314 cases from the Chinese Center for Disease Control and Prevention," Journal of the American Medical Association, vol. 323, no. 13, pp. 1239-1242, 2020.

[12] F. Fotouhi, M. Salehi-Vaziri, B. Farahmand et al., "Prolonged viral shedding and antibody persistence in patients with COVID-19," Microbes and Infection, vol. 23, no. 4-5, p. 104810, 2021.

[13] E. K. Z. L. Stokes, L. D. Zambrano, K. N. Anderson et al., "Coronavirus disease 2019 case surveillance - United States January 22-May 30, 2020," MMWR. Morbidity and Mortality Weekly Report, vol. 69, no. 24, pp. 759-765, 2020.

[14] World Health Organization, "Status of COVID-19 vaccines within WHO EUL/PQ evaluation process," 2021, https:// extranet.who.int/pqweb/sites/default/files/documents/Status_ COVID_VAX_11Nov2021.pdf.

[15] X. Liu and X.-J. Wang, "Potential inhibitors against 2019$\mathrm{nCoV}$ coronavirus $\mathrm{M}$ protease from clinically approved medicines," Journal of Genetics and Genomics, vol. 47, no. 2, pp. 119-121, 2020.

[16] F. Bessière, H. Roccia, A. Delinière et al., "Assessment of QT intervals in a case series of patients with coronavirus disease 2019 (COVID-19) infection treated with hydroxychloroquine alone or in combination with azithromycin in an intensive care unit," JAMA Cardiology, vol. 5, no. 9, pp. 1067-1069, 2020.
[17] S. Song, Y. Ji, G. Zhang et al., "Protective effect of atazanavir sulphate against pulmonary fibrosis in vivo and in vitro," Basic \& Clinical Pharmacology \& Toxicology, vol. 122, no. 2, pp. 199-207, 2018.

[18] F. Zhou, T. Yu, R. du et al., "Clinical course and risk factors for mortality of adult inpatients with COVID-19 in Wuhan, China: a retrospective cohort study," The Lancet., vol. 395, no. 10229, pp. 1054-1062, 2020.

[19] Y. Monteerarat, S. Sakabe, S. Ngamurulert et al., "Induction of TNF- $\alpha$ in human macrophages by avian and human influenza viruses," Archives of Virology, vol. 155, no. 8, pp. 1273-1279, 2010.

[20] E. Bissio and G. D. Lopardo, "Incidence of hyperbilirubinemia and jaundice due to atazanavir in a cohort of Hispanic patients," AIDS Research and Human Retroviruses, vol. 29, no. 3, pp. 415-417, 2013.

[21] C. R. Rathbun, M. D. Liedtke, S. M. Blevins et al., "Electrocardiogram abnormalities with atazanavir and lopinavir/ritonavir," HIV Clinical Trials, vol. 10, no. 5, pp. 328-336, 2009.

[22] E. Joyce, A. Fabre, and N. Mahon, "Hydroxychloroquine cardiotoxicity presenting as a rapidly evolving biventricular cardiomyopathy: key diagnostic features and literature review," European Heart Journal Acute Cardiovascular Care, vol. 2, no. 1, pp. 77-83, 2013.

[23] D. Bentué-Ferrer, C. Arvieux, O. Tribut, A. Ruffault, and E. Bellissant, "Clinical pharmacology, efficacy and safety of atazanavir: a review," Expert Opinion on Drug Metabolism \& Toxicology, vol. 5, no. 11, pp. 1455-1468, 2009.

[24] D. R. (Niel) Malan, E. Krantz, N. David, V. Wirtz, J. Hammond, and D. McGrath, "Efficacy and safety of atazanavir, with or without ritonavir, as part of once-daily highly active antiretroviral therapy regimens in antiretroviral-naive patients," Journal of Acquired Immune Deficiency Syndromes (1999), vol. 47, no. 2, pp. 161-167, 2008.

[25] C. Torti, G. Lapadula, A. Antinori et al., "Hyperbilirubinemia during atazanavir treatment in 2, 404 patients in the Italian atazanavir expanded access program and MASTER cohorts," Infection, vol. 37, no. 3, pp. 244-249, 2009.

[26] V. C. A. Russo, F. F. Mottola, R. Mocerino et al., "Effect of triple combination therapy with lopinavir-ritonavir, azithromycin, and hydroxychloroquine on QT interval and arrhythmic risk in hospitalized COVID-19 patients," Frontiers in Pharmacology, vol. 11, no. 582348, pp. 1-5, 2020. 